\title{
Errores y dificultades en la resolución de problemas algebraicos
}

\section{Errors and difficulties in solving algebraic problems}

\author{
Zulma consuelo Sánchez-García ${ }^{\mathrm{a}}$ \\ a* Magister en Educación Matemáticas, Colegio Integrado Campo Dos, 0000-0002-8285-336X, zulsy.zs.zs@gmail.com, , Cúcuta, \\ Colombia
}

Forma de citar: Sánchez García, Z.C. Errores y dificultades en la resolución de problemas algebraicos. Eco Matemático, 10 (2), 23-34

Recibido: 22 agosto 2019

Aceptado: 6 noviembre 2019

\section{Palabras clave \\ Errores, dificultades, problemas \\ algebraicos.}

\section{Keywords}

Errors, difficulties, algebraic problems.
Resumen: El estudio se desarrolló para determinar los errores y las dificultades que presentan los estudiantes del grado once de la Institución Educativa Misael Pastrana Borrero de Cúcuta al enfrentarse a la solución de problemas algebraicos. Se aplicó un diseño descriptivo con dos instrumentos. Los resultados permitieron detectar que, de los 12 errores que se tomaron como dimensiones de estudio, siguiendo a García (2010), se replican ocho, siendo el más significativo el error de cálculo simple. Se puede concluir que los errores que se evidencian, muy bien podrían ser producto de sus experiencias de aprendizaje que han tenido en sus estudios previos. Con respecto a las dificultades, la de mayor aparición fue la relacionada con los procesos de enseñanza.

Abstract: The study was developed to determine the errors and difficulties presented by the students of the eleventh grade of the Misael Pastrana Borrero Educational Institution of Cúcuta when facing the solution of algebraic problems. A descriptive design with two instruments was applied. The results allowed to detect that, of the 12 errors that were taken as study dimensions, following García (2010), eight are replicated, the most significant being the simple calculation error. It can be concluded that the errors that are evident, could very well be the product of their learning experiences that they have had in their previous studies. With respect to the difficulties, the one with the greatest appearance was related to the teaching processes.

*Autor para correspondencia zulsy.zs.zs@gmail.com 


\section{Introducción}

Diversas investigaciones en el contexto internacional, tal y como lo señalan Kaput y Blanton (2000), apoyan la introducción de contenidos algebraicos en el currículo de primaria y bachillerato. Sin embargo, esta misma diversidad, es una de las razones por la que tales investigaciones hayan tenido dificultades para su implantación. Al respecto, se reconoce que no se tiene una posición suficientemente explícita sobre la naturaleza del razonamiento algebraico en los niveles elementales de la educación primaria y media, ni una concepción de lo que se podría considerar como álgebra en estos niveles. Pero, si se tiene claro que, en el marco de la enseñanza de la Matemática, el álgebra es una disciplina importante, pues constituye un eje temático que contiene conocimientos imprescindibles para la vida educativa y social del estudiante.

Se podría decir con Socas (1997) que, gran parte de los estudios que se están desarrollando en la enseñanza de la Matemática, surgen del hecho observable de que el estudiante se equivoca cuando se le pide realizar ciertas actividades. Entonces, los investigadores en esta área del conocimiento estudian cuando el educando proporciona respuestas erróneas, con respecto a un patrón de evaluación, o simplemente no es capaz de dar ninguna respuesta; teniendo en cuenta que los errores y dificultades no se presentan de un modo aleatorio o imprevisible. La investigación en enseñanza de la matemática trata de caracterizar estas regularidades y de construir modelos explicativos, en términos de relaciones entre las variables, errores y dificultades.

En este sentido, Rico (1995) plantea que los errores y las dificultades forman parte de las producciones de los alumnos y constituyen, generalmente, dos elementos en los procesos de enseñanza y aprendizaje de la Matemática en todos los niveles del sistema educativo colombiano. De igual manera, si se considera que el correcto aprendizaje de la Matemática es un objetivo común en los procesos de enseñanza queda claro que las respuestas incorrectas a las cuestiones que se plantean a los estudiantes serán consideradas como indicadores de deficiencias.

Por ello, estudiar los errores que cometen y las dificultades que poseen los alumnos del grado once de la Institución Educativa Misael Pastrana Borrero de la ciudad de Cúcuta, Departamento Norte de Santander se constituye en el objetivo de esta investigación. De tal manera, que el estudio se sustenta en dos aportaciones teóricas: para la tipología de errores se sigue el estudio de García Suárez (2010) y para las dificultades se toma el enfoque de Di Blasi Regner y Otros (2003) citados por Abrate, Pochulu y Vargas (2006) por cuanto ambos fundamentos se adaptan a los requerimientos del autor del presente estudio. La metodología utilizada es cuantitativa, con un diseño descriptivo y para la recolección de datos el primer instrumento esta soportado y ajustado en algunas temáticas por las pruebas saber ICFES para detectar los errores, y se elaboró un instrumento con cinco preguntas para detectar las dificultades hacia la resolución de problemas algebraicos en los estudiantes del grado once.

\section{Marco teórico}

\section{Tipología del error, definicióny caracterización}

Según Gil (1997) el profesor debe ser sensible a las ideas previas de los alumnos. Al respecto, Orton (2003) y Gardner(1988) refieren que es bien conocido que, para muchos estudiantes, el álgebra resulta difícil y desde luego irrelevante, algunos llegan a experimentar un rechazo tan intenso que impregna el conjunto de su actitud hacia las matemáticas. Por ello, en el contexto de la clasificación de los errores, en esta investigación se toma la tipología presentada por García Suárez (2010: p.61) por cuanto se adapta a los objetivos de la misma. De tal manera, que se asume una clasificación con doce tipos de errores.

1) Eliminación incorrecta de denominadores. Este tipo de error el alumno reconoce que para 
eliminar un denominador debe multiplicar toda la expresión por un múltiplo del denominador, pero omite multiplicar todos los elementos de la misma, alterando de esta forma el resultado final.

2) Errores al realizar operaciones aritméticasalgebraicas. Dentro de esta clasificación se consiguen aquellas respuestas en las que aparentemente se sigue un procedimiento de resolución coherente, pero se comete un error al realizar alguna de las operaciones básicas aritméticas-algebraicas.

3) Procedimiento inconcluso. En algunas pruebas el procedimiento estaba parcialmente correcto, pero no se terminó.

4) Problemas propios incorrectos e inferencias no válidas. En algunas de las pruebas se identificaron errores en los cuales los alumnos utilizaban algún tipo de procedimiento en el que hacen inferencias no validas de reglas parcialmente recordadas, errores al transcribir datos, aplicación de métodos de tanteo y otras operaciones que aparentemente se realizan por el simple hecho de desarrollar algún procedimiento.

5) Aplicación parcial de regla de factorización por factor común. Este error se presenta cuando el alumno intenta separar los factores comunes, pero no recuerda el paso siguiente del procedimiento dejando inconcluso la operación o no verifica la validez del factor común, así como no respetar las reglas de los exponentes del citado factor.

6) Asociación incorrecta de productos notables. En este caso los alumnos intentan asociar las formas $\mathrm{y}$ fórmulas de productos notables para resolver la operación.

7) Uso de la aritmética básica ignorando las reglas del álgebra. El alumno intenta resolver la expresión algebraica como una operación aritmética, ya sea suma, resta o multiplicación de los coeficientes y exponentes de la misma.
8) Error en la determinación de la potencia de otra potencia. Este error se encuentra en algunas pruebas en las cuales el alumno desarrolla la fórmula para el binomio al cubo, pero se equivoca al aplicar la regla de multiplicación de los exponentes al parecer por una omisión al multiplicar los mismos.

9) Resolución aditiva de la potencia de un binomio. En estos casos los alumnos multiplican los exponentes de cada uno de los elementos de la expresión algebraica ignorando la formula correcta de resolución.

10) Aplicación incorrecta de la regla del cubo de un binomio. Este error se presenta cuando el estudiante intenta aplicar la formula correspondiente, pero al parecer no es capaz de recordarla de manera correcta, aunque en algunos casos los problemas posteriores tengan coherencia.

11) Error al realizar productos de polinomios. En este error el alumno descompone la expresión algebraica en factores simples, pero se equivoca al multiplicarlos para encontrar el resultado.

12) Error de cálculo simple. Este error se presenta cuando el alumno se equivoca al realizar alguna de las operaciones básicas de la aritmética y por lo tanto obtiene un valor incorrecto que sigue utilizando sin detectar el error.

\section{Definición, caracterización y tipología de las dificultades}

El término dificultad, en el ámbito pedagógico indica el mayor o menor grado de éxito de los estudiantes ante una actividad o tema de estudio. En este sentido, Palarea (1999) refiere que si el porcentaje de errores es elevado se dice que la dificultad es alta, mientras que, si dicho porcentaje es bajo, la dificultad es baja. Para el estudio de la tipología de las dificultades, se toma en consideración los postulados propuestos por Di Blasi Regner y Otros (2003) citados por Abrate, 
Pochulu y Vargas (2006, p.31) quienes las agrupan en los siguientes tópicos: a) dificultades asociadas a la complejidad de los objetos matemáticos, b) dificultades asociadas a los procesos de pensamiento matemático, c) dificultades asociadas a los procesos de enseñanza, dificultades asociadas al desarrollo cognitivo de los alumnos, dificultades asociadas a las actitudes afectivas y emocionales.

\section{La resolución de problemas como proceso matemático}

Alsina (2012) señala que, en los últimos tiempos, en las instituciones educativas, además de los bloques de contenido matemático, en los currículos actuales se ha empezado a dar protagonismo a los procesos matemáticos. Sepúlveda, Medina y Sepúlveda Jáuregui (2009) sobre la resolución de problemas plantean que el avance del conocimiento matemático se debe, en gran parte, a la resolución de los problemas que matemáticos, pero no es sino hasta los estudios de George Polya, en 1945, cuando esta actividad comienza a considerarse importante en la educación matemática.

En ese sentido, Polya y Zugazagoitia (1965) cuyas ideas fueron retomadas por Valiente (2000) establecen que la resolución de problemas es una característica esencial del ser humano. Por su parte, Torra (2014) y Kilpatrick, Rico y Sierra (1994) refieren que la resolución de problemas constituye una de las principales maneras de hacer matemáticas. En ese sentido, Kieran y Filloy (1989) y Alsina (2014) coinciden que en los procesos de resolución de problemas se deben utilizar estrategias metacognitivas que regulen el empleo eficaz de los recursos cognitivos y afectivos. Entre los aspectos a tomar en cuenta durante la resolución de problemas; Godino (2009), Coronata (2014), Castro (2008), Torra (2014), Pachón, Parada y Chaparro (2016) consideran establecer que, si el problema en realidad es un enunciado sin sentido, el nivel de dificultad del problema y el proceso de resolución es más complicado.

\section{Metodología}

La indagación desarrollada tuvo como soporte el paradigma cuantitativo con un diseño descriptivo, que de acuerdo con el punto de vista de Hernández Fernández y Baptista (2008, p.102) tiene como propósito "describir situaciones y eventos. La población estuvo conformada por dos grupos de 65 sujetos muéstrales. Para las técnicas e instrumentos se tomó como soporte, para elaborar el instrumento, las pruebas ICFES (2018) e ICFES (2017) referido al cuadernillo de preguntas Saber 11 y la guía de orientación Saber 11, ambos de la prueba de matemática. De estos cuadernillos se seleccionaron 12 preguntas que se organizaron en ítems de desarrollo, del cual fueron ajustados para el avance de la investigación. Además, se utilizó una entrevista con 5 preguntas que buscó recabar información sobre las dificultades que presentan los estudiantes del grado once de Institución Educativa Misael Pastrana Borrero al enfrentarse a la solución de problemas algebraicos.

\section{Resultados de la investigación}

En esta sección se presenta el análisis e interpretación de los resultados obtenidos producto de identificar los errores y las dificultades que presentan los estudiantes del grado once de la Institución Educativa Misael Pastrana Borrero, al enfrentarse a la solución de problemas algebraicos. Luego de aplicado los instrumentos, (prueba sobre lenguaje algebraico y entrevista) se procedió a presentar el análisis de la siguiente manera: a) primero se analizan los datos de la prueba de lenguaje algebraico donde se enfatiza en los errores y b) luego se analizan los datos de la entrevista para exponer las principales dificultades que presentan los estudiantes que participaron en el estudio. 
Análisis del cuestionario de los errores en procedimientos algebraicos en la resolución de problemas

Luego de analizados los errores del instrumento aplicado, se puede detectar que el error de mayor aparición, en la muestra de estudio, es el error de cálculo simple, seguido de los errores donde el estudiante deja el ejercicio sin culminar. Posteriormente, aparecen los errores sobre eliminación incorrecta de denominadores y errores al realizar operaciones aritméticas-algebraicas, seguido de problemas propios incorrectos e inferencias no validas, aplicación parcial de regla de factorización por factor común y uso de la aritmética básica ignorando las reglas del álgebra. Es importante destacar que surgieron tipos de errores no previstos por García Suarez (2010) como fueron: deficiencias en la conservación del área, valores de medida, y, además, olvido de los elementos matemáticos para resolver un problema.

Ahora bien, al revisar estos errores en los 12 ítems del instrumento se puede constatar que en el ítem 1 donde según el ICFES (2018) plantea que este ejercicio se refiere a la competencia argumentativa, la cual desarrolla un contenido de Álgebra y cálculo. El mismo, evalúa la capacidad del estudiante de encontrar argumentos que validan o refutan una afirmación hecha usando lenguaje algebraico. En los porcentajes de estudiantes que respondieron de manera incorrecta, cuando se revisó el procedimiento seguido por los participantes en la investigación, se observa que, mayoritariamente, se puede apreciar un porcentaje alto de similitud de errores con lo que plantea García Suárez (2010), particularmente surgieron dos tipos de errores: por un lado, surgió el error de cálculo simple y por otro, algunos estudiantes no concluyeron el ejercicio. Dentro de esta clasificación aparecen aquellas respuestas en las que se sigue un procedimiento de resolución coherente, pero se comete un error al realizar alguna de las operaciones básicas.
Por otra parte, en los resultados del ítem 2, se observa que los estudiantes que no respondieron correctamente $(43,1 \%)$ cometen, básicamente, el tipo de error planteado por García Suárez (2010) como error de cálculo simple. Es decir que se equivocan al realizar alguna de las operaciones básicas de la aritmética. Asimismo, al analizar la interrogante 3 se consigue un porcentaje de respuestas incorrectas relativamente alto con 75,4\%. Esta interrogante, según el ICFES (2018) tiene relación con argumentación de contenidos de Álgebra y Cálculo genérico y valida problemas y estrategias matemáticas utilizadas para dar solución a problemas. Por lo cual, la cantidad significativa de estudiantes que no acertaron la respuesta cometen algunos de los errores que plantea García Suárez (2010), referidos a errores al realizar operaciones aritméticas y algebraicas. Además, se aprecia en algunos estudiantes, el error de cálculo simple, problemas propios con inferencias no válidas y procedimiento inconcluso.

Revisando el procedimiento utilizado para resolver el ítem 4 se puede apreciar, con base en García Suárez (2010), tres tipos de errores que plantea este autor como el error denominado procedimiento inconcluso, pues se detectaron algunas pruebas en las cuales el procedimiento estaba parcialmente correcto pero no se terminó; por otro lado, en algunas pruebas se observó error de cálculo simple, dado que algunos estudiantes se equivocaron al realizar operaciones básicas de suma, multiplicación y división, y errores al realizar operaciones aritméticas algebraicas.

La interrogante 5 plantea un ítem del cual surgieron de acuerdo con los planteamientos de García Suárez (2010), tres tipos de errores referidos a problemas propios incorrectos e inferencias no válidas, error de cálculo simple y procedimiento inconcluso. Según el ICFES (2018) esta interrogante se refiere a la formulación y ejecución de un problema que involucra información cuantitativa, plantea 
estrategias que llevan a soluciones algebraicas adecuadas.

La interrogante 6 reflejó un porcentaje alto $(67,6 \%)$ de respuestas incorrectas. En este ítem, al echar una mirada a las distintas pruebas y siguiendo a García Suárez (2010), se aprecia un porcentaje alto de estudiantes que presentan cuatro tipos de errores: 1) Error de cálculo simple, surgió porque unos estudiantes se equivocan al realizar alguna de las operaciones básicas de la aritmética, 2) problemas propios incorrectos e inferencias no válidas, pues en algunas de las pruebas se identificaron errores en los cuales los alumnos utilizaban algún tipo de procedimiento en el que hacen inferencias no validas, errores al transcribir datos o aplicación de métodos de tanteo, 3) procedimiento inconcluso, dado que se detectaron algunas pruebas en las cuales el procedimiento estaba parcialmente correcto pero no se terminó y 4) errores al realizar operaciones aritméticas básicas.

En el ejercicio 7 el porcentaje más alto de participantes en la investigación $(56,9 \%)$ en esta interrogante cometen, mayoritariamente, el error que García Suárez (2010) refiere como error de cálculo simple, pues son varios los estudiantes que se equivocan al realizar alguna de las operaciones básicas de la aritmética. Además, se puede apreciar en este ítem que los estudiantes cometen el error de eliminar incorrectamente los denominadores o dejan el ejercicio sin culminar; además de utilizar la aritmética básica ignorando las reglas del álgebra.

Al analizar el ítem 8 el procedimiento utilizado en esta interrogante, se puede apreciar diversas maneras utilizadas por los estudiantes al desarrollar el ejercicio. Estas formas de trabajo conducen a dos errores típicos que se detectan en el porcentaje de respuestas incorrectas y que son planteados por García Suárez (2010): 1) Eliminación incorrecta de denominadores, donde en un gran número de estudiantes se aprecia que reconocen que para eliminar un denominador debe multiplicar toda la expresión por un múltiplo del denominador pero omiten multiplicar todos los elementos de la misma, alterando de esta forma el resultado final, 2) procedimiento inconcluso, en el cual los estudiantes no culminan el ejercicio, como se observa.

Al revisar el planteamiento del ítem 9, según el ICFES (2018) la respuesta correcta es 24 habitaciones pueden tener extensión telefónica, lo cual permite a los estudiantes plantear estrategias que lleven a soluciones adecuadas, respuesta que no fue acertada por un alto porcentaje de estudiantes. Esto significa que más de la mitad de los sujetos que participaron en la investigación presentan el error planteado por García Suárez (2010) como error de cálculo simple, dado que un porcentaje alto de estudiantes se equivocaron al realizar alguna de las operaciones básicas de la aritmética por lo cual utilizaron un valor incorrecto que siguieron utilizando sin detectar el error. Además, se apreció también, el error denominado procedimiento inconcluso, dado que algunos estudiantes dejaron el ejercicio sin culminar.

Al analizar el procedimiento utilizado en la interrogante 10 , se puede apreciar diversas maneras utilizadas por los estudiantes al desarrollar el ejercicio. Estas formas de trabajo conducen a dos errores típicos que se detectan en el porcentaje de respuestas incorrectas y que son planteados por García Suárez (2010): 1) Eliminación incorrecta de denominadores, donde en un gran número de estudiantes se aprecia que reconocen que para eliminar un denominador debe multiplicar toda la expresión por un múltiplo del denominador pero omiten multiplicar todos los elementos de la misma, alterando de esta forma el resultado final, 2) procedimiento inconcluso, en el cual los estudiantes no culminan el ejercicio, como se observa.

Al revisar el procedimiento utilizado por los estudiantes en el ítem 11 y relacionarlo con los planteamientos de García Suárez (2010) se puede decir, que en un porcentaje significativo de 
estudiantes cometieron errores al realizar operaciones aritméticas-algebraicas, pues se consiguió un alto porcentaje de respuestas en las que, aparentemente, se sigue un procedimiento de resolución coherente pero se comete un error al realizar alguna de las operaciones básicas aritméticas-algebraicas, tales como, multiplicación, reducción de términos semejantes, operaciones con números enteros, decimales o fracciones que incluyan variables. Entonces, al revisar el procedimiento.

Entonces, el ítem 12 plantea una interrogante sobre números racionales donde el mayor porcentaje no respondió correctamente la respuesta. En los problemas de los estudiantes que desarrollaron el procedimiento se consiguió, mayormente, errores sobre procedimiento inconcluso y error de cálculo simple. En cuanto a los problemas que se plantean al estudiante sobre la resolución de problemas con fracciones Flores, (2010) refiere que este constructo matemático, generalmente, ocasiona diversos problemas a los estudiantes, particularmente por una falta de formación en las nociones básicas de suma y resta. Pues la idea intuitiva de dividir una totalidad en partes iguales, donde a y b son números que están escritos uno sobre el otro, requiere una construcción de distintos significados.

\section{Análisis de la entrevista para conocer las dificultades de los estudiantes}

En el marco de las dificultades de los estudiantes de grado once se presenta un análisis a partir de haber revisado la entrevista. Entonces, revisada cada interrogante se presenta un análisis de las ideas de dos estudiantes seleccionados que permiten ampliar los diferentes errores que presentan, a partir de sus pensamientos sobre cuál o cuáles pueden ser las causas que generan los errores conseguidos. Estos dos estudiantes están codificados como Participante 1 (P1) y participante 2 (P2).

Entonces, al interrogar a los sujetos sobre las dificultades para resolver los planteamientos del ítem
1 en líneas resumidas se consiguieron las siguientes respuestas: uno de los participantes (P1) mencionó que "a veces se me olvida la multiplicación" y el sujeto 2 (P2) mencionó: "yo no logré comprender el ejercicio, pues a veces los problemas matemáticos se me olvidan fácilmente y sobre todo se me olvida las tablas" De tal modo que en términos generales, siguiendo a Di Blasi Regner y Otros (2003) citados por Abrate, Pochulu y Vargas (2006), se aprecian dificultades asociadas a los aspectos cognitivos del estudiante, lo cual hace que un alto porcentaje de ellos se le haga complicado la respuesta al ejercicio planteado.

Por otra parte, al preguntar a los estudiantes sobre las dificultades para resolver el ítem 2, se consiguieron distintas respuestas, relacionados con los métodos de enseñanza empleados en la matemática, los cuales, según uno de los participantes, (P2) no son los más adecuados para entender esta asignatura. Asimismo, este estudiante, manifiesta que siente ansiedad y miedo hacia las clases de matemática "porque siempre que tengo que desarrollar un ejercicio en matemática me produce algo en el estómago"

Los planteamientos del sujeto 2 (P2) están referidos a lo que Di Blasi Regner y Otros (2003) citados por Abrate, Pochulu y Vargas (2006) denominan dificultades asociadas a los procesos de enseñanza y a las actitudes afectivas y emocionales. Sobre, este aspecto se sabe que, a muchos estudiantes, incluyendo a algunos de los más capacitados, no les gusta la Matemática. Muchos discípulos tienen sentimientos de tensión y miedo hacia ella. Sin lugar a duda muchos son los aspectos que influyen en esta aversión. Siendo uno de ellos, los procesos de enseñanza los cuales deben reducir las dificultades del aprendizaje de la Matemática dependiendo de los materiales curriculares, de los recursos y de los estilos de enseñanza.

Con relación a los procesos de enseñanza de la matemática Cedillo (1999) acota que esta 
asignatura tiene conocimientos que no son nada fáciles de aprender, su aprendizaje requiere la creación de significados abstractos, la codificación y descodificación de símbolos y la capacidad de hacer relaciones en el plano de lo posible, por lo cual su enseñanza tiene que estar cargada de elementos de creatividad y de medios instruccionales novedosos para que los estudiantes participen activamente. Pues, como es conocido, el aprendizaje de las matemáticas es un aprendizaje complejo que debe acompañarse de buenas actitudes afectivas y emocionales que permita alcanzar un nivel de desarrollo cognitivo, que a su vez sustente los aprendizajes matemáticos.

En cuanto el componente afectivo se condensa siguiendo a Morales, Moya, Gaviria y Cuadrado (2007) quienes mencionan que la actitud afectiva se fundamenta en todas las impresiones del sujeto hacia la asignatura esencial, puntualmente cuando se trata de exámenes con valoración positiva o negativa. Este tipo de actitud lleva al estudiante a querer, a valorar y a plasmar un sentimiento de placer y ganas de aprender cualquier área de la malla curricular. Pero esto depende mucho del entusiasmo del docente por cuanto los diversos temas son dictados por ellos y son los profesores los que implantan las ganas por aprender o no con sus estrategias metodológicas y afectivas.

Las dificultades asociadas a los procesos de enseñanza tienen que ver con la institución escolar, con el currículo de Matemática y con los métodos de enseñanza. En tal sentido, Arrabal, Laporte y Rivas (2012) y Alsina, C. y otros (1996). mencionan que la institución escolar debe propiciar una organización escolar que tienda a reducir las dificultades del aprendizaje de la Matemática dependiendo de los materiales curriculares, de los recursos y de los estilos de enseñanza. Asimismo, las dificultades asociadas al desarrollo cognitivo de los alumnos, deben llevar a los docentes a revisar los estadios generales del desarrollo intelectual, representado cada uno de ellos por un modo característico de razonamiento y por unas actividades específicas de Matemática como es la enseñanza del cálculo simple.

Se puede decir que, en la transición de la aritmética al álgebra escolar, se presentan algunas dificultades que tienen los estudiantes al momento de aprender álgebra; por ello, la ideas de Godino (2003) son importantes, en tanto aborda algunas de las dificultades que presentan los estudiantes en el estudio del aprendizaje del álgebra, y plantea que durante los procesos de enseñanza, los profesores tienen que asumir que los niños y jóvenes requieren de prácticas innovadoras de enseñanza, sobre todo cuando se trata del aprendizaje de las operaciones básicas.

\section{Conclusiones}

El estudio sobre los errores y dificultades en la solución de resolución de problemas algebraicos en estudiantes del grado once de la institución educativa Misael Pastrana Borrero, deja algunas reflexiones en cuanto a los altos índices de errores conseguidos con el instrumento aplicado. Se podría pensar, de acuerdo a los aspectos teóricos mencionados, que los errores demostrados por los estudiantes durante el diagnóstico, se puede deber a la poca aplicación de estrategias correctas por parte de los docentes, pues, además, así lo manifestaron algunos estudiantes durante la entrevista. Igualmente, se piensa que la falta de ejecución de situaciones didácticas acordes a los nuevos tiempos, por parte del docente, representa una de las causas de la problemática planteada, donde se destaca, carencia de una planificación que permita ver los errores como elementos útiles para nuevos aprendizajes.

Con base en lo anterior, se puede reflexionar sobre el rol del docente en la enseñanza de la matemática. El cual, no necesariamente, debe ser un transmisor de conocimientos, sino un mediador entre sus estudiantes, las actividades y el conocimiento de manera que se pueda formar estudiantes con altos niveles de aprendizaje del álgebra. 
Con respeto al objetivo que permitió determinar los errores y las dificultades que presentan los estudiantes se puede decir que los errores tipitos que surgieron en la muestra están relacionados, mayormente, con el error de cálculo simple, seguido de los errores donde el estudiante deja el ejercicio con el procedimiento inconcluso, luego aparece errores al realizar operaciones aritméticas-algebraicas, seguido eliminación incorrecta de denominadores, problemas propios incorrectos e inferencias no validas, aplicación parcial de regla de factorización por factor común y uso de la aritmética básica ignorando las reglas del álgebra. Es importante destacar que surgieron tipos de errores no previstos por García Suarez (2010) como fue el olvido de los elementos matemáticos para resolver un problema.

En líneas generales, y con base en la muestra, los estudiantes cometen un conjunto de errores en la solución de problemas algebraicos. A partir de los resultados obtenidos, con la aplicación del instrumento a los estudiantes, es posible inferir que los errores que se evidencian, muy bien podrían ser producto de sus experiencias de aprendizaje que han tenido en sus estudios previos. Los errores son notados en las actividades de los alumnos, en especial cuando se enfrentan a conocimientos que los obligan a hacer una revisión o reestructuración de lo que ya saben.

Con respecto a las dificultades, la de mayor aparición fue la relacionada con los procesos de enseñanza. En este sentido, el docente del área de matemáticas debe jugar un rol activo en el aprendizaje y comprender la finalidad que los estudiantes aprendan de manera significativa, es decir las clases deben ser creativas, dinámicas, activas, productivas y participativas. El aprendizaje es más eficaz cuando los estudiantes saben que sus errores servirán para nuevos aprendizajes, eso les puede llevar a demostrar atracción y gusto por el área de las matemáticas, emprendiendo una actividad común valiéndose de verdaderos instrumentos metodológicos para afianzar sus conocimientos.
Se puede profundizar el estudio de las dificultades que tienen los alumnos en el aprendizaje de los problemas algebraicos, en especial, buscando nuevas formas de observar de los errores.

Por otra parte, de manera general, ante la compleja realidad de la enseñanza de la resolución de problemas algebraicos ya no es posible por parte de los docentes seguir pensando y actuando desde una perspectiva reducida caracterizada por la búsqueda de recetas para mejorar el proceso de enseñanza y de aprendizaje del álgebra. Al respecto, Dubois (2011) enfatiza que los profesores necesitan un conocimiento profesional o mejoramiento en la enseñanza ya que su responsabilidad es realizar su práctica docente de manera adecuada, particularmente en la didáctica de los problemas algebraicos.

Asimismo, al concluir este estudio, se ha llegado a la conclusión que una buena alternativa para iniciar este proceso de mejoramiento, es afectar las prácticas de los docentes a través del uso de estrategias novedosas. Pues, el rol del docente en la enseñanza, particularmente en cuanto al error y la corrección, no necesariamente debe ser un transmisor de conocimientos, sino un mediador entre sus estudiantes, las actividades y el conocimiento de manera que los errores sean vistos como fuentes de aprendizaje.

Como investigadora principiante, este trabajo, que se inició hace más de un año, deja muchos aprendizajes. A nivel teórico se conoció distintos investigadores que han profundizado en los tipos de errores que cometen los estudiantes cuando resuelven problemas algebraicos. En lo metodológico deja una estela de preguntas para seguir el camino de los aprendizajes y en lo posible continuar en esta línea de investigación, que, cuando se inició, no se tenía pensado el enorme bagaje de conocimientos que se podrían adquirir. 
Las conclusiones permiten algunas recomendaciones reflexiones finales. $\mathrm{Al}$ respecto el objetivo de los problemas planteados en el aula para la enseñanza de la matemática $\mathrm{y}$, particularmente, de los problemas algebraicos; debe ser el descubrimiento de nuevos conocimientos y deben formularse en un lenguaje claro y familiar para el alumno, es decir conectado con sus experiencias vividas. Es importante, en estos tiempos y muy aconsejable, ayudarse de materiales instruccionales tecnológicos.

Algunas pautas recomendadas para la enseñanza de problemas algebraicos que minimicen los errores, están referidas a que los profesores deben proponer problemas con enunciados claros y concisos para facilitar la comprensión. Es muy importante tener en cuenta el grado de dificultad que lleva cada problema que, depende de su estructura semántica y del lugar que ocupe la incógnita. Una recomendación interesante está relacionada con ayudar a los alumnos a que visualicen los problemas y ejercicios matemáticos y darles el tiempo que sea necesario para la adecuada comprensión.

Desde el Currículo Oficial se insiste en la necesidad de que los estudiantes de educación secundaria vean el área de matemáticas como una disciplina imprescindible para la vida diaria y que entiendan que muchos problemas y situaciones reales no tienen solución sin un determinado conocimiento matemático. Sin embargo, es una de las disciplinas que más dificultades conlleva entre los estudiantes. Por ello, el papel fundamental de los docentes es hacer de la enseñanza de la matemática un tema agradable y lleno de retos para el estudiante.

Este estudio, apoyado en las investigaciones de referencia, da cuenta de los errores en este campo y la necesidad por construir marcos teóricos generales desde los cuales puedan ser tratados sistemáticamente. Parece más acertado establecer marcos referidos a un contenido temático curricular y determinar, no sólo una clasificación, sino una explicación de su origen, al menos a nivel individual. Los hallazgos dan cuenta de las propuestas por profundizar en las dificultades y errores que tienen los alumnos en los procesos necesarios para el razonamiento algébrico.

Asimismo, es importante reflexionar sobre la necesidad de impulsar actividades de intercambio entre los docentes, de tal modo que se promueva el establecimiento de círculos de estudio, orientados a la unificación de ideas en el área de matemática. Además, se requiere diseñar estrategias vinculadas con mecanismos que permitan contribuir con un mejor aprendizaje de los problemas algebraicos, sobre todo buscando una didáctica que promueva el uso de la tecnología de información y comunicación como herramienta de apoyo.

\section{Referencias}

Abrate, Raquel S., Pochulu, Marcel D., Vargas, José M. (2006). Errores y dificultades en Matemática. Análisis de causas y sugerencias de trabajo. Córdoba: Universidad Nacional de Villa María. Alsina, Á. (2012). Más allá de los contenidos, los procesos matemáticos en Educación Infantil. Edma 0-6: Educación Matemática en la Infancia, $1(1), 1-14$.

Alsina, A. y Coronata, C. (2014). Los procesos matemáticos en las prácticas docentes: diseño, construcción y validación de un instrumento de evaluación. Edma 0-6: Educación Matemática en la Infancia, 3(2), 23-36. •23•

Alsina, C. y otros (1996). Enseñar Matemáticas. Barcelona: Grao

Arrabal, M., Laporte, E, y Rivas, S. (2012). Una reflexión sobre la importancia del error y su corrección en la clase de español como L2.

Castro, E. (2008). Didáctica de la matemática en Educación Primaria. Madrid: Síntesis, S.A.

Cedillo, T. (1999). Nubes de puntos y modelación algebraica. México: Iberoamérica.

Coronata, C. (2014). Presencia de los procesos matemáticos en la enseñanza del número de 4 a 8 años. Transición entre la Educación Infantil y 
Elemental. Tesis doctoral. Girona: Universidad de Girona. Disponible en: http://dugi-doc.udg. edu/handle/10256/9750

Dubois, M. E. (2011). La lectura en la formación y Actualización del docente. Comentario sobre dos experiencias. Legenda, Vol. 15 nro. 12, enero-junio. Recuperado de: http://erevistas. saber.ula.ve/index.php/legenda/article/view/733

García Suárez, J. (2010). Análisis de errores $y$ dificultades en la resolución de tareas algebraicas por alumnos de primer ingreso en nivel licenciatura. Universidad de Granada. Facultad de Ciencias de la Educación. Recuperado de: https://fqm193.ugr.es/media/ grupos/FQM193/cms/Jose_Garcia.pdf

Gardner, M. (1988). Matemáticas para divertirse. Buenos Aires: Juan Granica.

Gil, D. (1997). Formación del profesorado de las Ciencias y la matemática. Madrid: Editorial Popular S.A.

Godino, J. (2003). Razonamiento algebraico para maestros. Recuperado de: http:/www.ugr.es/ local/jgodino/edumatmaestros/.

Godino, J.D. (2009). Categorías de análisis de los conocimientos del profesor de matemáticas. UNION, Revista Iberoamericana de Educación Matemática, 20, 13-31.

Hernández, R., Fernández, C., y Baptista, P. (2008) Metodología de la Investigación. México: McGrawHill.

Instituto Colombiano para la Evaluación de la Educación (ICFES) (2018). Cuadernillo de preguntas Saber 11. Prueba de Matemáticas. Recuperadode:www.icfes.gov.co/docman/...y.../ saber...informe...resultados-saber...2016/file

Instituto Colombiano para la Evaluación de la Educación (ICFES) (2017). Guía de orientación. Saber 11. Prueba de Matemáticas. Recuperado de: $\quad$ www.icfes.gov.co/docman/...y.../saber... informe...resultados-saber...2016/file

Kaput, J., y Blanton, M. L. (2000). El razonamiento algebraico en el contexto de la matemática elemental: su implementación en una escala masiva. Reunión anual de la Asociación de
Investigación Educativa de América del Norte, Montreal, Canadá.

Kieran, C, y Filloy Yague, E (1989). El aprendizaje del álgebra escolar desde una perspectiva psicológica. Enseñanza de las ciencias, 7 (3), 229240. Recuperado de: https://www.raco.cat/index. php/ensenanza/article/viewFile/51268/93013.

Kilpatrick, J.; Rico, L. y Sierra, M. (1994). Educación matemática e investigación. Madrid: Síntesis.

Morales, J. F., Moya, M., Gaviria, E. y Cuadrado, I. (2007). Psicología social. Madrid: Mc GrawHill.

Orton, A. (2003) Didáctica de las matemáticas. Madrid: Morata.

Pachón, L. Parada, R., y Chaparro, A. (2016) El razonamiento como eje transversal en la construcción del pensamiento lógico. Praxis \& Saber, 7(14), 219 - 143. Recuperado de: https:// doi.org/10.19053/22160159.5224

Palarea, M. (1999). La adquisición del lenguaje algebraico: reflexiones de una investigación. Revista de didáctica de las Matemáticas. Vol 40, diciembre, pp 3-28. Disponible en: http://www. sinewton.org/numeros/numeros/40/Articulo01. pdf

Polya, G., y Zugazagoitia, J. (1965). Cómo plantear y resolver problemas. Trillas. Recuperadodehttps://scholar.google.es $/ \mathrm{r} ? \mathrm{q}=\mathrm{c} \%$ $\mathrm{C} 3 \% \mathrm{~B} 3 \mathrm{mo}+$ plantear $+\mathrm{y}+$ resolver + problemas $+\mathrm{p}$ oly a\&hl $=$ es\&as_sdt $=0 \% 2 \mathrm{C} 5 \& \mathrm{oq}=\mathrm{como}+$ plant

Rico, L. (1995). Errores y dificultades en el aprendizaje de las Matemáticas. En Kilpatrik, J.; Gómez, P., Y Rico, L.: Educación Matemática. México: Grupo Editorial Iberoamérica.

Sepúlveda, A., Medina, C. y Sepúlveda, D. (2009). La resolución de problemas y el uso de tareas en la enseñanza de las matemáticas. Educación matemática, 21(2), 79-115. Recuperado en 04 de abril de 2019, de http://www.scielo.org. $\mathrm{mx} /$ scielo.php? script $=$ sci_arttext\&pid $=\mathrm{S} 1665$ 58262009000200004\&lng=es\&tlng=es.

Socas, M. (1997). Dificultades, obstáculos y errores en el aprendizaje de las Matemáticas en la 
Educación Secundaria. En: Rico, L. (1997). La educación matemática en la enseñanza secundaria. Barcelona: ICE/Horsori.

Torra, M. (2014). Indicadores competenciales: un instrumento para la mejora del desarrollo de la competencia matemática. Edma 0-6: Educación Matemática en la Infancia, 3(1), 81-86.

Valiente, S. (2000). Didáctica de la matemática. El libro de los recursos. Madrid: La Muralla S. A. 